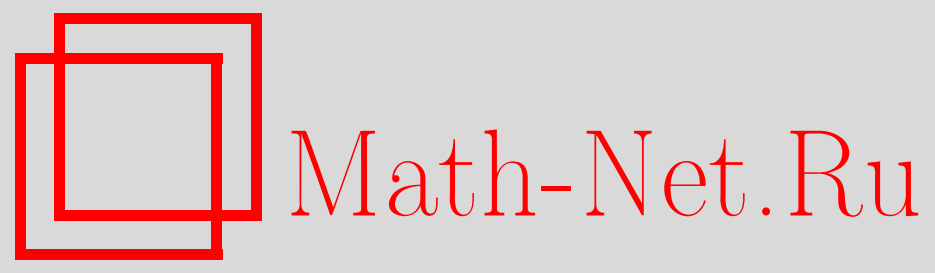

С. Н. Селезнева, О сложности представления функций многозначных логик поляризованными полиномами, Дискрет. матем., 2002, том 14, выпуск 2, 48-53

DOI: https://doi.org/10.4213/dm240

Использование Общероссийского математического портала Math-Net.Ru подразумевает, что вы прочитали и согласны с пользовательским соглашением http://www . mathnet.ru/rus/agreement

Параметры загрузки:

IP : 35.173 .219 .149

26 апреля 2023 г., 10:26:58 
УдК 519.7

\title{
О сложности представления функций многозначных логик поляризованными полиномами
}

\author{
() 2002 г. С. Н. Селезнева
}

\begin{abstract}
Понятие поляризованной полиномиальной формы обобщается на случай функций многозначных логик. Вводятся функции Шеннона веса и длины поляризованных полиномиальных форм функций многозначных логик и доказываются некоторые их оценки.

Работа выполнена при поддержке Российского фонда фундаментальных исследований, грант 00-01-00351.
\end{abstract}

\section{1. Введение}

В алгебре логики рассматривается представление функций поляризованными полиномиальными формами, которые также называются обобщенными формами Рида-Маллера, или каноническими поляризованными полиномами $[1,2]$. Пусть

$$
\sigma=\left(\sigma_{1}, \ldots, \sigma_{n}\right)
$$

- набор элементов из множества $E_{2}=\{0,1\}$. Поляризованной полиномиальной формой по вектору поляризации $\sigma$ называется полином относительно операций суммы по модулю 2 и умножения, в котором каждая переменная $x_{i}$ встречается как $x_{i}^{\sigma_{i}}, i=1, \ldots, n$. Длиной поляризованной полиномиальной формы называется число ее слагаемых. Для каждой булевой функции $f\left(x_{1}, \ldots, x_{n}\right)$ ее представление полиномиальной поляризованной формой по каждому вектору поляризации однозначно. Обозначим его $P^{\sigma}(f)$. Для исследования сложности представления булевых функций поляризованными полиномиальными формами вводится функция Шеннона. Функция Шеннона длины $L(n)$ определяется как $\max \min l\left(P^{\sigma}(f)\right)$, где $l\left(P^{\sigma}(f)\right)$ - длина поляризованной полиномиальной формы $P^{\sigma}(f)$, минимум берется по всем возможным векторам поляризации, максимум - по всем функциям, зависящим от $n$ переменных.

Н. А. Перязевым в [3] получена точная оценка функции Шеннона

$$
L(n)=\left[(2 / 3) 2^{n}\right]
$$

(выражение $[a]$ обозначает целую часть числа $a$ ).

В настоящей работе понятие поляризованной полиномиальной формы обобщается на случай функщий многозначных логик и исследуется сложность полученного представления функций многозначных логик. 


\section{2. Основные понятия}

Пусть $k$ - простое число, $k \geqslant 3, P_{k}$ - множество всех функщий $k$-значной логики $f^{n}: E_{k}^{n} \rightarrow E_{k}$, где $E_{k}=\{0,1, \ldots, k-1\}$. Обозначим $S_{k}$ множество всех перестановок из $P_{k}$, то есть одноместных функщий, принимающих все $k$ значений.

Назовем вектором поляризации $\varphi$ произвольный набор $\left(\varphi_{1}(x), \ldots, \varphi_{n}(x)\right)$ функщий из $S_{k}$. Поляризованной полиномиальной формой, или поляризованным полиномом по вектору поляризации $\varphi$ назовем выражение вида

$$
\sum_{i=1}^{l} X_{i}
$$

в котором каждое слагаемое $X_{i}$ имеет вид

$$
c_{i}\left(\varphi_{i_{1}}\left(x_{i_{1}}\right)\right)^{m_{i_{1}}} \ldots\left(\varphi_{i_{r_{i}}}\left(x_{i_{r_{i}}}\right)\right)^{m_{i r_{i}}}, \quad c_{i} \in E_{k},
$$

и называется поляризованным одночленом, сумма и произведение рассматриваются по модулю $k$. Полагается, что в поляризованных полиномах приведены все подобные слагаемые.

Теорема 1. Для произвольного вектора поляризачии ч для любой функции $f\left(x_{1}, \ldots, x_{n}\right)$ из $P_{k}$ существует единственная ее поляризованная полиномиальная форма по вектору $\varphi$.

Доказательство. Все функщии $\varphi_{i}(x)$ принадлежат множеству $S_{k}$, поэтому существуют обратные к ним функщии $\varphi_{i}^{-1}(x), i=1, \ldots, n$.

Рассмотрим функцию $g\left(x_{1}, \ldots, x_{n}\right)=f\left(\varphi_{1}^{-1}\left(x_{1}\right), \ldots, \varphi_{n}^{-1}\left(x_{n}\right)\right)$. Так как $k-$ простое число, каждая функщия из $P_{k}$ представима полиномом по модулю $k$ [4]. Пусть

$$
g\left(x_{1}, \ldots, x_{n}\right)=\sum_{i=1}^{l} c_{i} x_{i_{1}}^{m_{i_{1}}} \ldots x_{i_{r_{i}}}^{m_{i_{r_{i}}}}
$$

Тогда

$$
f\left(x_{1}, \ldots, x_{n}\right)=\sum_{i=1}^{l} c_{i}\left(\varphi_{i_{1}}\left(x_{i_{1}}\right)\right)^{m_{i_{1}}} \ldots\left(\varphi_{i_{r_{i}}}\left(x_{i_{r_{i}}}\right)\right)^{m_{i_{r_{i}}}} .
$$

Единственность представления следует из единственности представления функции $k$-значной логики полиномом по модулю $k$.

Теорема доказана.

Поляризованный полином по вектору поляризации $\varphi$ функщии $f\left(x_{1}, \ldots, x_{n}\right)$ будем обозначать $P^{\varphi}(f)$.

Введем понятия сложности поляризованных полиномов. Типом выражения

$$
c_{i}\left(\varphi_{i_{1}}\left(x_{i_{1}}\right)\right)^{m_{i_{1}}} \ldots\left(\varphi_{i_{r_{i}}}\left(x_{i_{r_{i}}}\right)\right)^{m_{r_{i}}}
$$

назовем множество $\left\{\varphi_{i_{1}}\left(x_{i_{1}}\right), \ldots, \varphi_{i_{r_{i}}}\left(x_{i_{r_{i}}}\right)\right\}$. Весом поляризованного полинома $P$ назовем число попарно различных типов его слагаемых и обозначим его $w(P)$. Длиной поляризованного полинома $P$ назовем число его слагаемых и обозначим его $l(P)$. Заметим, что $w(P) \leqslant l(P)$ для произвольного поляризованного полинома $P$. В случае двузначной логики понятия веса и длины поляризованных полиномов совпадают. 
Пусть $A \subseteq S_{k}$. Рассмотрим функции Шеннона сложностей представления функций $k$-значной логики полиномами, поляризованными функщиями из множества $A$. Функцию Шеннона веса поляризованных полиномов $W_{A}(n)$ определим следующим образом: пусть

$$
W_{A}(f)=\min w\left(P^{\varphi}(f)\right),
$$

где минимум берется по всевозможным векторам поляризации $\varphi$, составленным из функций множества $A$, тогда

$$
W_{A}(n)=\max W_{A}(f),
$$

где максимум берется по всем функциям $f$, зависящим от $n$ аргументов.

Аналогично для функщии Шеннона длины поляризованных полиномов $L_{A}(n)$ :

$$
L_{A}(f)=\min l\left(P^{\varphi}(f)\right),
$$

где минимум берется по всевозможным векторам поляризации $\varphi$, составленным из функций множества $A$, и

$$
\left.L_{A}(n)=\max w_{A}(f)\right),
$$

где максимум берется по всем функциям $f$, зависящим от $n$ аргументов.

\section{3. Некоторые оценки функций Шеннона}

Теорема 2. Для любого множества $A, A \subseteq S_{k}, A \neq \varnothing$, справедливо равенство $W_{A}(n)=2^{n}$.

Доказательство. Рассмотрим функцию

$$
g(x)=x^{k-1}+1
$$

Докажем от противного, что $W_{A}(g)=2$. Предположим, что найдется такая функция $\varphi(x)$ в множестве $A$, что

$$
g(x)=c_{k-1}(\varphi(x))^{k-1}+c_{k-2}(\varphi(x))^{k-2}+\ldots+c_{1} \varphi(x),
$$

где $c_{k-1}, c_{k-2}, \ldots, c_{1} \in E_{k}$. Так как $\varphi(x) \in S_{k}$, найдется такой элемент $a$ из $E_{k}$, что $\varphi(a)=0$. Из представления (2) следует, что $g(a)=0$ и из представления (1) следует, что $g(a)=2$ (или $g(a)=1$, если $a=0$ ). Получаем противоречие при $k \geqslant 3$. Следовательно, в любом поляризованном полиноме функции $g(x)$ присутствует ненулевой свободный член. С другой стороны, функция $g(x)$ не является константой, поэтому любой ее поляризованный полином содержит еще хотя бы одно слагаемое. Таким образом, $W_{A}(g)=2$.

Рассмотрим функцию

$$
f\left(x_{1}, \ldots, x_{n}\right)=\left(x_{1}^{k-1}+1\right)\left(x_{2}^{k-1}+1\right) \ldots\left(x_{n}^{k-1}+1\right) .
$$

Из доказанного выше следует, что $W_{A}(f)=2^{n}$. Всего же существует $2^{n}$ типов слагаемых поляризованных полиномов функций, зависящих от $n$ аргументов. Следовательно, $W_{A}(n)=2^{n}$.

Теорема доказана.

Следствие 1. Для любого множества $A, A \subseteq S_{k}, A \neq \varnothing$, справедливо неравенство $L_{A}(n) \geqslant 2^{n}$. 
Теорема 3. Для множества $A, A=\{x, x+1, x+2, \ldots, x+(k-1)\}$, справедливо неравенство

$$
L_{A}(n)<\frac{k(k-1)}{k(k-1)+1} k^{n} .
$$

Доказательство. Доказательство проведем индукщией по числу $n$. Некоторые идеи индуктивного перехода почерпнуты из [3].

В качестве базиса индукщии возьмем случай $n=1$. Рассмотрим произвольную функцию $f(x)$. Пусть ее полином по модулю $k$ имеет вид

$$
c_{k-1} x^{k-1}+\ldots+c_{1} x+c_{0}
$$

где $c_{k-1}, \ldots, c_{1}, c_{0} \in E_{k}$. Если $c_{k-1}=0$, то в качестве поляризованного полинома функции $f(x)$ оставим ее полином по модулю $k$. Его длина не больше $k-1$. В противном случае выполним следующие преобразования: вынося за скобку множитель $c_{k-1}$, получаем выражение

$$
f(x)=c_{k-1}\left(x^{k-1}+\frac{c_{k-2}}{c_{k-1}} x^{k-2}+\ldots+\frac{c_{1}}{c_{k-1}} x+\frac{c_{0}}{c_{k-1}}\right)
$$

в выражении, оставшемся в скобках, выделим слагаемые, образующие разложение выражения $(x+d)^{k-1}$, где элемент $d$ выбирается так, чтобы поглотить слагаемое $c_{k-2} x^{k-2}$, в результате получаем выражение

$$
\begin{aligned}
f(x) & =c_{k-1}\left(x^{k-1}+(k-1) \frac{c_{k-2}}{c_{k-1}(k-1)} x^{k-2}+\ldots\right) \\
& =c_{k-1}(x+d)^{k-1}+c_{k-3}^{\prime} x^{k-3}+\ldots+c_{1}^{\prime} x+c_{0}^{\prime},
\end{aligned}
$$

где

$$
d=\frac{c_{k-2}}{c_{k-1}(k-1)}
$$

Представим функцию $f(x)$ полиномом, поляризованным по вектору $\varphi=(x+d)$. Его длина будет не больше $k-1$.

Утверждение теоремы в этом случае справедливо, так как

$$
l\left(P^{\varphi}(f)\right)=k-1<\frac{k(k-1)}{k(k-1)+1} k=\frac{(k(k-1)+1)-1}{k(k-1)+1} k=k-\frac{k}{k(k-1)+1} .
$$

Проведем индуктивный переход. Предположим, что теорема верна при некотором значении $n$. Докажем, что в таком случае теорема также верна и при значении $n+1$.

Рассмотрим произвольную функщию $f\left(x_{1}, \ldots, x_{n}, x_{n+1}\right)$. Запишем ее полином по модулю $k$ в виде

$x_{n+1}^{k-1} g_{k-1}\left(x_{1}, \ldots, x_{n}\right)+x_{n+1}^{k-2} g_{k-2}\left(x_{1}, \ldots, x_{n}\right)+\ldots+x_{n+1} g_{1}\left(x_{1}, \ldots, x_{n}\right)+g_{0}\left(x_{1}, \ldots, x_{n}\right)$.

По предположению индукщии существует поляризованный полином функции $g_{k-1}\left(x_{1}, \ldots, x_{n}\right)$, длина которого меньше $k^{n}(k(k-1)) /(k(k-1)+1)$. Пусть $\varphi=\left(x+d_{1}, \ldots, x+d_{n}\right)-$ его вектор поляризации.

Запишем каждую из функций

$$
g_{k-1}\left(x_{1}, \ldots, x_{n}\right), g_{k-2}\left(x_{1}, \ldots, x_{n}\right), \ldots, g_{1}\left(x_{1}, \ldots, x_{n}\right), g_{0}\left(x_{1}, \ldots, x_{n}\right)
$$


полиномом, поляризованным по вектору $\varphi$. В результате получим полином функции $f\left(x_{1}, \ldots, x_{n}, x_{n+1}\right)$, поляризованный по вектору $\psi=\left(x+d_{1}, \ldots, x+d_{n}, x\right)$.

Представим его в виде

$$
\sum_{X} X f_{X}\left(x_{n+1}\right)
$$

где $X$ пробегает все возможные одночлены, составленные из переменных $x_{1}, \ldots, x_{n}$ и поляризованные по вектору $\varphi$. Для каждого полинома $f_{X}\left(x_{n+1}\right)$ выполним преобразования, описанные в базисе индукщии. А именно, пусть полином $f_{X}\left(x_{n+1}\right)$ имеет вид $c_{k-1} x_{n+1}^{k-1}+\ldots+c_{1} x_{n+1}+c_{0}$, где $c_{k-1}, \ldots, c_{1}, c_{0} \in E_{k}$. Если $c_{k-1}=0$, то оставляем его без изменений. В противном случае приведем его к виду

$$
c_{k-1}\left(x_{n+1}+d\right)^{k-1}+c_{k-3}^{\prime} x_{n+1}^{k-3}+\ldots+c_{1}^{\prime} \ldots x_{n+1}+c_{0}^{\prime},
$$

где

$$
d=\frac{c_{k-2}}{c_{k-1}(k-1)}
$$

В зависимости от значения коэффищиента $c_{k-1}$ полином $f_{X}\left(x_{n+1}\right)$ перепишем либо в виде

$$
c_{k-1}\left(x_{n+1}+d\right)^{k-1}+f_{X}^{\prime}\left(x_{n+1}\right),
$$

либо оставим без изменения в виде $c_{k-2} x_{n+1}^{k-2}+f_{X}^{\prime \prime}\left(x_{n+1}\right)$ или $f_{X}^{\prime \prime \prime}\left(x_{n+1}\right)$. Заметим, что в полиномах $f_{X}^{\prime}\left(x_{n+1}\right), f_{X}^{\prime \prime}\left(x_{n+1}\right)$ и $f_{X}^{\prime \prime \prime}\left(x_{n+1}\right)$ не встречаются слагаемые степени выше, чем $k-3$.

Таким образом, поляризованный полином функции $f\left(x_{1}, \ldots, x_{n}, x_{n+1}\right)$ можно записать как

$$
\begin{aligned}
x_{n+1}^{k-1} g_{k-1}^{(0)}\left(x_{1}, \ldots, x_{n}\right) & +\left(x_{n+1}+1\right)^{k-1} g_{k-1}^{(1)}\left(x_{1}, \ldots, x_{n}\right)+\ldots \\
& +\left(x_{n+1}+(k-1)\right)^{k-1} g_{k-1}^{(k-1)}\left(x_{1}, \ldots, x_{n}\right) \\
& +x_{n+1}^{k-2} g_{k-2}\left(x_{1}, \ldots, x_{n}\right)+h\left(x_{1}, \ldots, x_{n}, x_{n+1}\right)
\end{aligned}
$$

где в полиноме $h\left(x_{1}, \ldots, x_{n}, x_{n+1}\right)$ переменная $x_{n+1}$ имеет степени не выше, чем $k-3$. Заметим, что

$$
g_{k-1}\left(x_{1}, \ldots, x_{n}\right)=g_{k-1}^{(0)}\left(x_{1}, \ldots, x_{n}\right)+g_{k-1}^{(1)}\left(x_{1}, \ldots, x_{n}\right)+\ldots+g_{k-1}^{(k-1)}\left(x_{1}, \ldots, x_{n}\right) .
$$

Важно также отметить, что любые два из поляризованных полиномов

$$
g_{k-1}^{(0)}\left(x_{1}, \ldots, x_{n}\right), g_{k-1}^{(1)}\left(x_{1}, \ldots, x_{n}\right), \ldots, g_{k-1}^{(k-1)}\left(x_{1}, \ldots, x_{n}\right), g_{k-2}\left(x_{1}, \ldots, x_{n}\right)
$$

не содержат совпадающих слагаемых.

Выберем такой элемент $i$, что длина поляризованного полинома

$$
\sum_{s \neq i} g_{k-1}^{(s)}\left(x_{1}, \ldots, x_{n}\right)
$$

меньше

$$
\frac{k-1}{k} \frac{k(k-1)}{k(k-1)+1} k^{n}
$$


Такой элемент $i$ всегда найдется. Построим полином функции $f\left(x_{1}, \ldots, x_{n}, x_{n+1}\right)$, поляризованный по вектору $\xi=\left(x+d_{1}, \ldots, x+d_{n}, x+i\right)$, для чего каждую скобку вида $(x+d)^{k-1}$ в выражении (3) запишем как

$$
((x+i)+(d-i))^{k-1}
$$

и раскроем по формуле бинома Ньютона. Тогда функцию $f\left(x_{1}, \ldots, x_{n}, x_{n+1}\right)$ можно представить в виде

$$
\left(x_{n+1}+i\right)^{k-1} I_{1}+\left(x_{n+1}+i\right)^{k-2} I_{2}+I_{3}+\left(x_{n+1}+i\right)^{k-3} J_{k-3}+\ldots+J_{0},
$$

где

$$
\begin{aligned}
& I_{1}=g_{k-1}\left(x_{1}, \ldots, x_{n}\right), \quad I_{2}=\sum_{s \neq i} c_{s} g_{k-1}^{(s)}\left(x_{1}, \ldots, x_{n}\right), \quad I_{3}=g_{k-2}\left(x_{1}, \ldots, x_{n}\right), \\
& J_{k-3}=h_{k-3}\left(x_{1}, \ldots, x_{n}\right), \quad \ldots, \quad J_{1}=h_{1}\left(x_{1}, \ldots, x_{n}\right), \quad J_{0}=h_{0}\left(x_{1}, \ldots, x_{n}\right),
\end{aligned}
$$

$c_{s}$ - некоторые коэффициенты, $s \neq i$. Оценим длину представления. Ясно, что сумма длин частей $I_{1}$ и $I_{3}$ не больше $k^{n}$, длина части $I_{2}$ меньше

$$
\frac{k-1}{k} \frac{k(k-1)}{k(k-1)+1} k^{n}
$$

в силу выбора элемента $i$, длина каждой части $J_{0}, J_{1}, \ldots, J_{k-3}$ не больше $k^{n}$.

Таким образом,

$$
\begin{aligned}
l\left(P^{\varphi}(f)\right) & <\frac{k-1}{k} \frac{k(k-1)}{k(k-1)+1} k^{n}+(k-1) k^{n}=(k-1) k^{n}\left(\frac{k-1}{k(k-1)+1}+1\right) \\
& =(k-1) k^{n} \frac{k-1+k^{2}-k+1}{k(k-1)+1}=\frac{k(k-1)}{k(k-1)+1} k^{n+1} .
\end{aligned}
$$

Теорема доказана.

\section{Список литературы}

1. Sasao T., Besslich P., On the complexity of mod-2 sum PLA's. IEEE Trans. Comput. (1990) 39, №2, 262-266.

2. Супрун В. П., Сложность булевых функций в классе канонических поляризованных полиномов. Дискретная математика (1993) 5, №2, 111-115.

3. Перязев Н. А., Сложность булевых функций в классе полиномиальных поляризованных форм. Алгебра и логика (1995) 34, №3, 323-326.

4. Яблонский С. В., Функциональные построения в $k$-значной логике. Труды Матем. ин-та им. В. А. Стеклова АН СССР (1958) 51, 5-142. 